\title{
VOLTAGE SECURITY ENHANCEMENT USING ENERGY BASED SENSITIVITIES
}

\author{
Thomas J. Overbye \\ Student Member, IEEE \\ Christopher L. DeMarco \\ Member, IEEE \\ Department of Electrical and Computer Engineering \\ University of Wisconsin-Madison \\ Madison, WI 53706
}

\begin{abstract}
This paper uses an energy based security measure to define vulnerability to voltage instability, and proposes a method of security enhancement by examining the sensitivity of the security margin to various control actions. The use of the energy function in systems with significant line losses is reviewed, and the analytic expressions for first order total derivatives with respect to relevant control parameters derived. Computational properties, such as sparsity of various vector and matrix quantities, are examined. Numerical examples of security enhancement based on these sensitivities are computed for a thirty bus example system. Potential application of this approach within a security constrained optimal power flow is also discussed.
\end{abstract}

KEYWORDS: Voltage stability, voltage collapse, security enhancement, energy functions, optimal power flow.

\section{Introduction and Background}

Recent reports indicate that voltage instability is becoming an increasingly common problem for many utilities [1], [2]. In many cases, while the system may reach a vulnerable state through an equipment outage or earlier contingency, the immediate cause of voltage instability is not a large disturbance to the system. Rather the system operating point gradually changes over a period of minutes to hours from a state of relative security to one where collapse is imminent. Thus the longer time frame of the problem allows manual operator intervention in addition to automatic control actions. To take the most effective preventive control actions, an accurate, easily computable indicator of the proximity of the system to voltage collapse is needed.

In present day security-constrained optimal power flow, voltage security is assessed via bus voltage magnitude limits [3]. However, voltage instability problems have been shown to occur in systems where voltage magnitudes never decline below acceptable levels [4]. Furthermore, near the point of collapse voltage variations can be extremely sensitive to changes in load [5], so knowledge of the voltage level only at the current operating point may not be sufficient to predict the onset of stability problems. The need to expand the optimal power flow problem to include voltage security is recognized in [3] and [6].

90 SM 478-8 PWRS A paper recommended and approved by the IEEE Power System Engineering Comittee of the IEEE Power Engineering Soclety for presentation at the IEEE/PES 1990 Summer Meeting, Minneapolis, Minnesota, July 15-19, 1990. Manuscript submitted August 3, 1989; made available for printing May 18, 1990.
Recently a large number of papers have addressed the issue of quantifying the "distance" of a particular operating point to voltage collapse (typically in terms of allowable range of system parameter changes). Crucial in judging the effectiveness of a given security measure is that it is physically meaningful, varies smoothly to changes in the system (i.e. does not exhibit discontinuous changes in value for small system changes), and alerts operators and planners to the necessity and type of control actions. In [7] the relevant system parameters are assumed to move along a given "path" (i.e., the vector of parameters is assumed to be a known function of time), until the point of voltage collapse is reached. The time to reach the collapse point then used to rank the vulnerability of different system configurations to voltage collapse. Numerous authors have recognized that the Jacobian of the powerflow becomes singular at the point of voltage collapse [5],[8],[9], using this as the indicator that the collapse point has been reached. In [9] and [10] the smallest singular value of the Jacobian is proposed as a qualitative measure of proximity to voltage collapse. In [11] and [12] indices which attempt to quantify the distance between a pair of multiple powerflow solutions are presented.

The goal of this work is to apply the energy based measure presented by the authors in [13] to increase the security of a power system. The paper is organized as follows. First, the application of energy methods to the voltage collapse problem is briefly reviewed. Next sensitivities based upon the closed form energy function found in [14] are derived. The application of these sensitivities to improve system security is then demonstrated on a 5 bus system and the New England 30 bus system. Lastly, the computational issues of the method are discussed.

\section{Energy Based Security Measure}

For completeness the application of energy based security measures to power system voltage instability problem is briefly reviewed. In [14] a security measure was proposed to indicate vulnerability to voltage collapse based upon Lyapunov's direct method. Formally, the term Lyapunov function is used to refer to a scalar function of state that has the properties of being locally positive definite about a stable equilibrium and non-increasing along trajectories. For the models used in this paper, in which the second property can not be guaranteed to hold for all instants in time, the scalar function of state will be referred to as an energy function. Application of the energy function to the problem of voltage insecurity is as follows. The current operating state of the power system (normally available on-line via the state estimator) can be thought of as defining a local minimum of an energy well. However, the true instantaneous state may not sit precisely at the mathematically defined equilibrium point, because it is constantly being perturbed by small, random variations (e.g. changing loads). These small magnitude random load variations add a small 
amount of kinetic "energy" to the system. This energy is normally dissipated through damping in the system. At a secure operating point, these small random effects are negligible, and are typically not considered in normal power systems analysis. However, under the conditions associated with proximity to collapse, the system state (particularly voltage magnitudes) are highly sensitive to load changes. Under such conditions, there is a possibility that these random perturbations could push the state out of the potential well that defines its stable operating point. A necessary condition for the state to escape this well is that it receives energy greater than the potential value of the closest unstable equilibrium point (UEP) on the boundary of the well. In the voltage stability context these UEPs or saddle points correspond to the alternative solutions of the powerflow equations [15],[16] that typically display very low voltage magnitudes at one or more buses. These will be referred to as the low voltage power flow solutions.

The use of energy functions to calculate proximity to voltage collapse is somewhat different from their use in transient stability. In the latter context, a fault or other large disturbance is applied to the power system, causing the state to rapidly move away from its former stable equilibrium point. Using the simplest Lyapunov based criterion, if the initial energy following the fault is less than that of the UEP with the lowest energy, then the system will asymptotically return to the post-fault equilibrium point. Other more sophisticated criteria make use of such concepts as the "controlling UEP" or "potential energy boundary surface. " These approaches recognize that a fault which yields a system trajectory passing exactly through the lowest saddle point is a rare, worst case scenario.

However, as noted in the introduction, the voltage collapse problem often occurs long after the power system has seemingly "settled down" following a large disturbance, or may occur without any large disturbance initiating the event. Rather, the system is generally subjected to a slow (time scale of minutes to hours) variation caused by the gradual changes in the in load/generation injections. Therefore the operating point of the system moves in a "quasi-static" manner. Of course small, random variations mentioned earlier are constantly perturbing the system away from this equilibrium. As indicated above, for a normal, robust operating point the energy well is quite deep, so these random perturbations have a negligible effect. To determine the significance of these random variations, one needs information about the height of the "easiest" path of escape out of the potential well; i.e. the energy necessary to exit through the lowest saddle point or closest u.e.p. This height can be measured by calculating the energy difference between the appropriate low voltage solution and the current state, and serves as the security measure that will be used to indicate relative vulnerability to voltage problems. As the system moves closer to the point of voltage collapse, work in [13] demonstrated many examples in which the energy difference decreases in an almost linear manner with system changes. The near linear variation of the energy measure with system changes is demonstrated in Figure 1 using the New England 30 Bus system from [17] as the reactive load at bus 11 is increased. In this scenario once the height of the energy well is sufficiently small, the ultimate collapse is driven by the small system perturbations mentioned earlier. Once the state leaves the potential well about the operating point, the deterministic dynamics drive a very rapid decline in voltage magnitudes. From a system security point of view, it is certainly desirable to use the available system controls to increase this energy difference and thus prevent the small perturbations from dominating and ultimately causing voltage collapse. The longer time scale of this problem allows for the application of security enhancement type software. The goal of this work is to develop a methodology for identifying the most effective control actions to increase this energy difference.

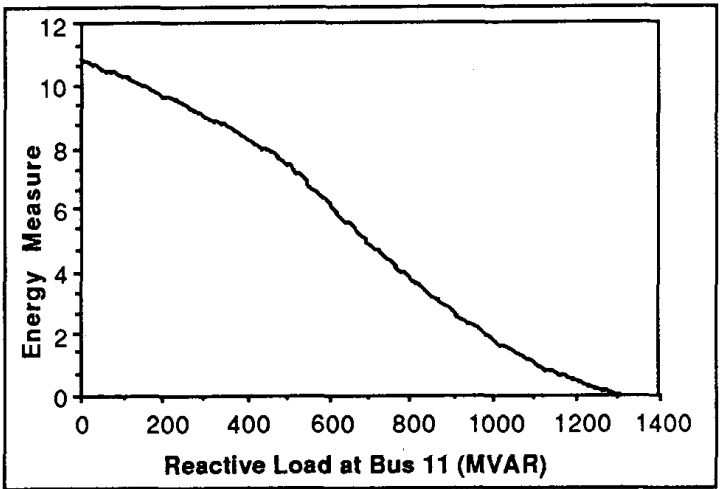

FIGURE 1: Energy Based Security Measure versus Bus Loading

For a system model in which transfer conductances are neglected, the energy function used above for measuring the height of the potential barrier between the unstable and stable equilibrium points is defined as follows:

$$
\vartheta\left(\mathbf{x}^{\mathbf{s}}, \mathbf{x}^{\mathbf{u}}\right)=\int_{\left(\alpha^{s}, \mathbf{v}^{\mathbf{s}}\right)}^{\left(\alpha^{\mathrm{u}}, \mathbf{v}^{\mathrm{u}}\right)}\left[\mathbf{f}^{\mathrm{T}}(\xi, v), \mathbf{g}^{\mathrm{T}}(\xi, v)\right]^{\mathrm{T}}[\mathrm{d} \xi, \mathrm{dv}]^{\mathrm{T}}
$$

where

$$
\begin{aligned}
& \mathbf{x}^{\mathbf{s}}:=\text { Stable equilibrium point }=\left(\alpha^{\mathbf{s}}, \mathbf{v}^{\mathbf{s}}\right) \\
& \mathbf{x}^{\mathbf{u}}:=\text { Unstable equilibrium point }=\left(\alpha^{\mathbf{u}}, \mathbf{v}^{\mathbf{v}}\right) \\
& f_{i}(\alpha, v)=P_{i}-\sum_{j=1}^{n} B_{i j}\left|v_{i}\right|\left|v_{j}\right| \sin \left(\alpha_{i}-\alpha_{j}\right) \\
& g_{i}(\alpha, v)=\left(V_{i}\right)^{-1}\left(Q_{i}+\sum_{j=1}^{n} B_{i j}\left|v_{i}\right|\left|V_{j}\right| \cos \left(\alpha_{i}-\alpha_{j}\right)\right)
\end{aligned}
$$

Note that (2) is simply the real power mismatch equations, and the (3) the reactive power mismatch equations (multiplied by $\mathrm{V}^{-1}$ ), where both expressions neglect transfer conductances. An energy function with structure similar to this was presented in [18]. Moreover, since the energy function is constructed as an integral, the fundamental theorem of calculus assures that differentiating with respect to either the upper of lower limit will simply return \pm the function under the integral; in this case, the vector of power mismatch equations. Therefore, the partial derivative of the energy function with respect to its first or second argument will evaluate to zero at an equilibrium point, because mismatch must be zero at an equilibrium. In [14], it is shown that for a lossless system $\vartheta$ is formally a Lyapunov function, and thus it is not surprising that it has a local minimum, and hence zero first derivative, at $\mathbf{x}^{\mathbf{s}}$. However, if the system model itself does contain losses, $\alpha^{\mathrm{s}}, \mathrm{V}^{\mathrm{s}}$ no longer defines a local minimum of the 
$\vartheta$ function as defined above, and the first derivative of $\vartheta$ at $\left(\alpha^{s}, V^{s}\right)$ is no longer identically zero. This difficulty can be resolved at the stable operating point $\left(\alpha^{s}, V^{s}\right)$ by redefining $f$ and $\mathbf{g}$ used in (1) as follows:

$$
\begin{aligned}
& f_{i}(\alpha, V)=P_{i}-\sum_{j=1}^{n} B_{i j}\left|v_{i}\right|\left|v_{j}\right| \sin \left(\alpha_{i}-\alpha_{j}\right) \\
& -\sum_{j=1}^{n} G_{i j}\left|v_{i}^{s}\right|\left|v_{j}^{s}\right| \cos \left(\alpha_{i}^{s}-\alpha_{j}^{s}\right) \\
& g_{i}(\alpha, V)=\left(V_{i}\right)^{-1}\left(Q_{i}+\sum_{j=1}^{n} B_{i j}\left|v_{i}\right|\left|v_{j}\right| \cos \left(\alpha_{i}-\alpha_{j}\right)\right) \\
& -\left(V_{i}^{s}\right)^{-1} \sum_{j=1}^{n} G_{i j}\left|v_{i}^{s}\right|\left|v_{j}^{s}\right| \sin \left(\alpha_{i}^{s}-\alpha_{j}^{s}\right) .
\end{aligned}
$$

Since the added terms in (4), (5) relative to (2), (3) are constants with respect to the variable of integration, the vector function remains exactly integrable (i.e., no path dependence). However, with the addition of these constant offset terms, the gradient of the energy function at the stable equilibrium is now identically zero. The revised form of the integral defined in (1) can be expressed in closed form as:

$$
\begin{aligned}
& \vartheta\left(x^{s}, x\right)=-\frac{1}{2} \sum_{i=1}^{n} \sum_{j=1}^{n} B_{i j}\left|v_{i}\right|\left|v_{j}\right| \cos \left(\alpha_{i}-\alpha_{j}\right) \\
& +\frac{1}{2} \sum_{i=1}^{n} \sum_{j=1}^{n} B_{i j}\left|v_{i}^{s}\right|\left|v_{j}^{s}\right| \cos \left(\alpha_{i}^{s}-\alpha_{j}^{s}\right) \\
& -\left(\sum_{i=1}^{n} \int_{v_{i}^{s}}^{v_{i}} \frac{Q_{j}(x)}{x} d x\right)-P^{T}\left(\alpha-\alpha^{s}\right) \\
& -\sum_{i=1}^{n}\left(\sum_{j=1}^{n} G_{i j}\left|v_{i}^{s}\right|\left|v_{j}^{s}\right| \cos \left(\alpha_{i}^{s}-\alpha_{j}^{s}\right)\left(\alpha_{i}-\alpha_{i}^{s}\right)\right) \\
& -\sum_{i=1}^{n}\left(\left(V_{i}^{s}\right)^{-1} \sum_{j=1}^{n} G_{i j}\left|v_{i}^{s}\right|\left|v_{i}^{s}\right| \sin \left(\alpha_{i}^{s}-\alpha_{j}^{s}\right)\left(V_{i}-V_{i}^{s}\right)\right.
\end{aligned}
$$

For load buses the integral term in (6) can be quite easily evaluated when the reactive load is an arbitrary polynomial or exponential function of bus voltage. For example if the reactive load at bus $i$ is constant then the integral evaluates to

$$
\mathrm{Q}_{\mathrm{i}} * \ln \left(\frac{\mathrm{V}_{\mathrm{i}}^{\mathrm{u}}}{\mathrm{V}_{\mathrm{i}}^{\mathrm{s}}}\right)
$$

In the powerflow calculations used in this paper, the reactive output of regulating generator buses is assumed to vary in order to hold its bus voltage within a small tolerance of a given voltage setpoint. Exciter saturation is modeled by representing the reactive output of the generator as a function of terminal voltage, with saturation to specified upper and lower limits. With this model, the mathematical framework for treating var sources is identical to that for voltage dependent reactive loads. A typical var output versus terminal voltage characteristic is shown in figure 2 . If the reactive output of the generator has not yet reached a limit at either the high and low voltage solutions, the deviation from setpoint is very small, and this integral term is negligible. In the more common case when reactive output is pushed to a limit at the low voltage solution and the generator is still regulating in the high voltage solution, the integral term is well approximated by:

$$
\mathrm{Q}_{\lim (\max , \min )} * \ln \left(\frac{\mathrm{v}^{\mathrm{u}}}{\mathrm{v}^{\mathrm{s}}}\right)
$$

where at least one of $\mathrm{V}^{\mathrm{u}}$ or $\mathrm{V}^{\mathrm{s}}$ is at either its maximum or minimum reactive limit. The rationale for this is that along the integration path the reactive output of the generator would rapidly saturate once the voltage has moved outside the small tolerance about its setpoint (figure 2), and thus may be considered as a constant. Note that in this case (8) is the same as (7). This is to be expected since the generator bus behaves as a PQ bus along almost all of the path of integration.

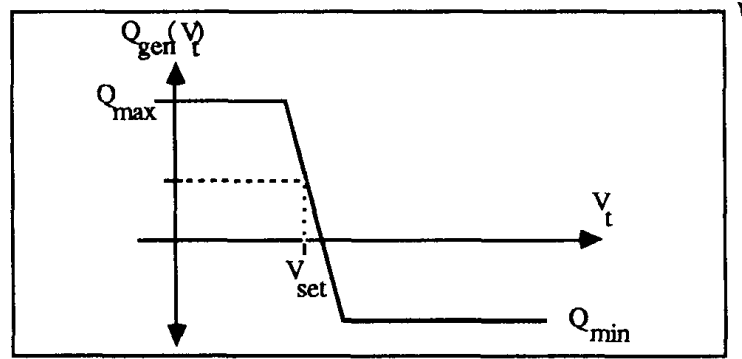

FIGURE 2: Generator Reactive Output versus Terminal Voltage

Thus an advantage of the energy function method is that limits on available var support can be taken into account, even when the current system operating point has not yet pushed generators to their limits. Intuitively this is because the low voltage solutions are characterized by large reactive flows which tend to push adjacent var sources to their limits. Likewise other types of voltage dependent automatic control actions (such as transformer tap movement) could also be included in the energy function.

\section{Analytic Derivation of Energy \\ Based Controller Sensitivities}

The availability of a closed form expression of the energy function facilitates the derivation of controller sensitivities through a Taylor expansion. However, since the energy function is inherently a function of both the current and low voltage state, it should be emphasized that these values are not simply linearizations about the current operating point. Similar analytic sensitivities have been previously derived by authors working with the transient stability energy function. In [19] numerous simulations of actual power systems have shown that such first 
order sensitivities can often be successfully used to improve system transient stability, though under some circumstances, second order terms from the Taylor expansion must be considered to obtain acceptable accuracy. In this section the sensitivity to controls of the voltage stability energy function are derived.

The variables used in (6) can be partitioned as follows:

$\mathbf{x}^{\mathrm{S}}, \mathbf{x}^{\mathbf{u}}$ - State variables (voltages and phase angles at all buses except for the slack) at stable and unstable equilibrium points;

u - Control variables (generator real power output, set point voltage, tap positions, etc.);

p - Uncontrolled parameters

The parameter dependence of the energy function can then be displayed as:

$$
\vartheta\left(\mathbf{u}, \mathbf{p}, \mathbf{x}^{\mathbf{s}}(\mathbf{u}), \mathbf{x}^{\mathbf{u}}(\mathbf{u})\right)
$$

For the derivation to follow, it is important to stress that the control parameters enter the energy function both explicitly and implicitly; the implicit dependence comes through motion of the equilibria under the effect of controller changes. With this observation in mind, first order sensitivities of the energy function with respect to changes in the control variables are calculated by simple application of the chain rule

$$
\begin{aligned}
& \text { d } \vartheta / \text { du }:=\text { Total Derivative } \vartheta\left(\mathbf{u}, \mathbf{p}, \mathbf{x}^{\mathbf{s}}(\mathbf{u}), \mathbf{x}^{\mathbf{u}}(\mathbf{u})\right) \text { w.r.t. } \mathbf{u} \\
& =\left(\partial \vartheta / \partial \mathbf{u}+\partial \vartheta / \partial \mathbf{x}^{\mathbf{s}} \cdot \partial \mathbf{x}^{\mathbf{s}} / \partial \mathbf{u}+\partial \vartheta / \partial \mathbf{x}^{\mathbf{u}} \cdot \partial \mathbf{x}^{\mathbf{u}} / \partial \mathbf{u}\right)
\end{aligned}
$$

If the system model is such that $\vartheta$ is formally a Lyapunov function for the system equations (i.e., if the system equations neglect losses), one would expect that $\vartheta$ would have a zero first derivative with respect to the state variables at all equilibria. Under these conditions, the second and third terms in the above expression would be identically zero. The reader should recall from the discussion above that this is not the case when losses are considered. Moreover, as with all linearizations, error between the linearized approximation and the exact energy variation depends upon the magnitude of the higher order terms and of the range of variation of the control variables.

Sensitivities could then be derived for any of the following types of controls: generator MW/Mvar/voltage setpoint, load MW/Mvar, transformer tap, MW transaction, phase shifter tap, and shunt capacitance. Computationally, the cost to calculate each controller sensitivity is on the order of a forward and backward substitution of the powerflow equations. For illustrative purposes, the sensitivity equations for changes in generator real power output (MW) is examined in the following example. This example will consider the influence of MW output on a generator's peak Mvar capacity, denoted below as $\mathrm{Q}\left(\mathrm{P}_{\mathrm{Gj}}\right)_{\text {limit }}$

With $x=[\alpha,|\mathbf{V}|]^{T}$ the sensitivity of energy with respect to generator real power output at bus $j$ is given by

$$
\begin{aligned}
& \partial \vartheta / \partial \mathrm{P}_{\mathrm{Gj}}=\left(\alpha_{j}^{\mathrm{u}}-\alpha_{j}^{\mathrm{s}}\right)+ \\
& {\left[\partial \vartheta / \partial \mathbf{x}^{\mathrm{u}}\right]^{\mathrm{T}} \partial \mathbf{x}^{\mathrm{u}} / \partial \mathrm{P}_{\mathrm{Gj}}+\left[\partial \vartheta / \partial \mathbf{x}^{\mathrm{s}}\right]^{\mathrm{T}} \partial \mathbf{x}^{\mathrm{s}} / \partial \mathrm{P}_{\mathrm{Gj}}+}
\end{aligned}
$$

$$
\begin{aligned}
& \left(\left[\partial \vartheta / \partial \mathbf{x}^{\mathrm{u}}\right]^{\mathrm{T}} \partial \mathbf{x}^{\mathrm{u}} / \partial \mathrm{Q}\left(\mathrm{P}_{\mathrm{Gj}}\right)_{\text {limit }}+\left[\partial \vartheta / \partial \mathbf{x}^{\mathrm{s}}\right]^{\mathrm{T}} \partial \mathrm{x}^{\mathrm{s}} / \partial \mathrm{Q}\left(\mathrm{P}_{\mathrm{Gj}}\right)_{\text {limit }}\right) \\
& \partial \mathrm{Q}\left(\mathrm{P}_{\mathrm{Gj}}\right)_{\text {limit }} / \partial \mathrm{P}_{\mathrm{Gj}}
\end{aligned}
$$

Note that (11) can be quite efficiently calculated since the terms $\partial \vartheta / \partial \mathbf{x}^{\mathrm{u}}$ and $\partial \vartheta / \partial \mathbf{x}^{\mathbf{s}}$ depend only upon first neighbors of generator $j$, and the terms $\partial \mathrm{x}^{\mathrm{u}} / \partial \mathrm{P}_{\mathrm{Gj}}, \partial \mathrm{x}^{\mathrm{s}} / \partial \mathrm{P}_{\mathrm{Gj}}, \partial \mathbf{x}^{\mathrm{u}} / \partial \mathrm{Q}\left(\mathrm{P}_{\mathrm{Gj}}\right)_{\text {limit }}$, and $\partial \mathbf{x}^{\mathrm{s}} / \partial \mathrm{Q}\left(\mathrm{P}_{\mathbf{G}_{\mathrm{j}}}\right)_{\text {limit }}$ can be determined using sparse vector methods [20]. As noted above, the last term is included to account for the variation in the unit's reactive power limits with respect to changes in real power output. Since systems vulnerable to voltage instability are often characterized by a number of saturated units, the effect of re-dispatching the real power on the unit's reactive capability must be considered. Scenarios where system security is enhanced by backing down the real power output of a saturated unit in order to increase its reactive capability can thus be properly modeled.

\section{Application to Test Systems}

The use of the control sensitivities previously derived is demonstrated in this section first on a 5 bus system and then on the New England 30 bus test system. The aim of this section is to demonstrate that system voltage security at a given operating point can be "maximized" by moving controllers to maximize the energy measure (difference). This approach is provided for illustrative purposes; in an application environment tradeoffs between economy and security would normally be required. This maximization is accomplished through the following algorithm:

0) Start from a given network state (normally available on-line from the state estimator).

1) Calculate the appropriate low voltage solution (see Section $\mathrm{V}$ for a description of the algorithms used).

2) Calculate the controller sensitivities.

3) Use a gradient search to move controllers in the direction of increasing energy measure.

4) Energy measure maximum is reached when all controller sensitivities are zero (unless a controller is constrained at its limit); otherwise Goto step 1.

A rough measure of the effectiveness of the energy method can be obtained by comparing the amount of load increase the original and the re-dispatched systems can withstand before voltage collapse occurs.

The Stagg and El-Abiad 5 bus system [21] was chosen as the first test system with bus 1 as the slack. The system was slightly modified so that the bus 2 generator's voltage regulation point was set to 1.04 per unit, and that its reactive capability was modeled using the piecewise linear curve shown in figure 3 . 


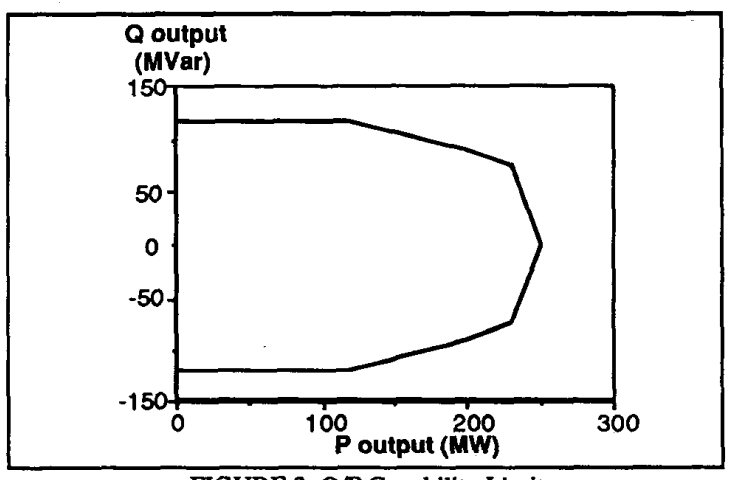

FIGURE 3: Q/P Capability Limits

For this example all real and reactive loads are assumed to be linear functions of the parameter $\mathbf{k}$ which will be referred to as the load constant ( $k=1$ for basecase); $P_{G 2}$ is initially assumed fixed at $40 \mathrm{MW}$. Hence increasing $k$ parameterizes the total system load increase, and for a sufficiently high $k$ the point of Jacobian singularity is eventually reached, guaranteeing voltage collapse. With no controller movement this occurs when $k=3.27$. The lower curve in figure 4 plots the energy measure vs $k$, with an energy value of zero corresponding to the point of voltage collapse where the high and low voltage solutions coalesce.

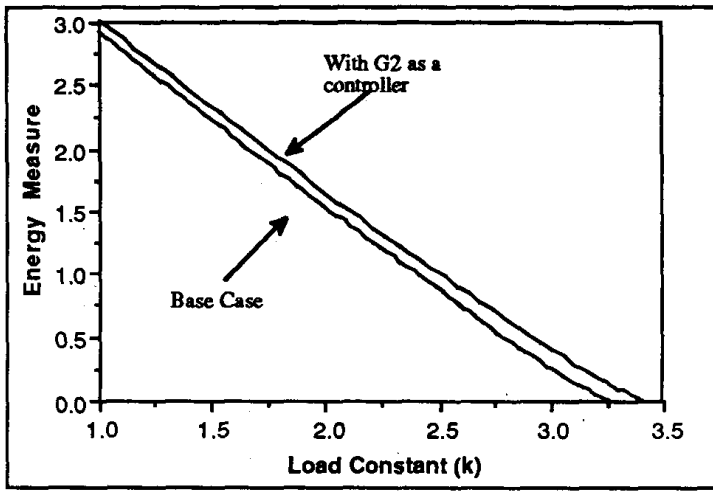

FIGURE 4: Energy Measure Improvement with Redispatch

Now assume that a single variable, the MW output of $G_{2}$ is available for control. Thus at any load level the sensitivity of the energy measure to $P_{G 2}$ can be calculated by (11). Anytime this value is nonzero it is possible to increase the system energy (at least incrementally) by changing the $M W$ output of $G_{2}$, and there by decrease the system's vulnerability to voltage collapse. The validity of the sensitivity is of course dependent upon the size of the neighborhood in which the energy measure can be approximated by a linear function of the controls. Figure 5 , which plots the variation in the system energy measure as $P_{G 2}$ is increased, shows that for $\mathrm{k}=1$ the system energy is indeed a nearly linear function of $P_{G 2}$ up to the breakpoints in the generator's reactive capability curve. This breakpoint dependence is expected because of the explicit dependence in (11) of $\partial \vartheta / \partial P_{\mathrm{Gj}}$ upon $\partial \mathrm{Q}\left(\mathrm{P}_{\mathrm{Gj}}\right)_{\text {limil }} / \partial \mathrm{P}_{\mathrm{Gj}}$ (figure 3).

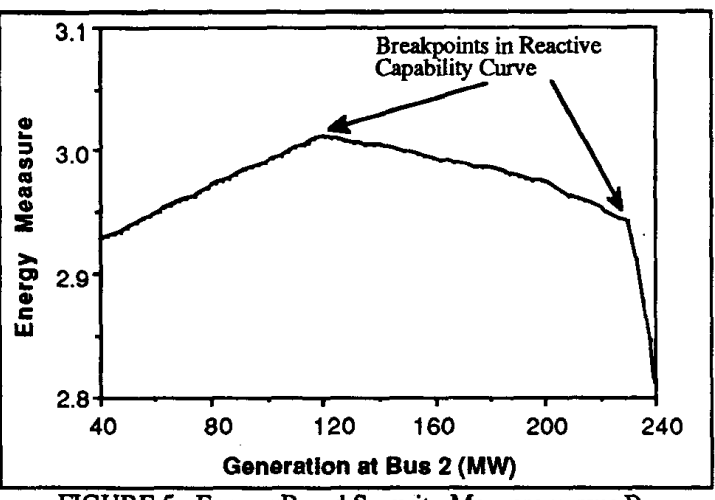

FIGURE 5: Energy Based Security Measure versus $\mathrm{P}_{\mathrm{C} 2}$

For $\mathrm{k}=1$ and $\mathrm{PG} 2=40 \mathrm{MW}, \partial \vartheta / \partial \mathrm{P}_{\mathrm{G} 2}=0.118$ per unit which indicates that the energy measure can be increased by increasing the $M W$ output of $G_{2}$. The energy maximum, which can be found by using a gradient search algorithm, occurs when $P_{G 2}=120 \mathrm{MW}$. This value is not unsurprising since it represents the breakpoint in the reactive capability curve (figure 3 ) where further increases in real power output result in a decrease in reactive capability. As the system load changes over time subsequent optimizations could be performed periodically to insure that the system stays at its energy maximum. The upper curve in figure 4 plots these maximum energy values versus $k$. Note that the system is now able to tolerate a maximum load corresponding to $\mathrm{k}=3.42$ before voltage collapse occurs. The "optimal" $\mathrm{P}_{\mathrm{G} 2}$ at this point is equal to 202MW. Stated another way, the objective is to maximize the amount of load the system can tolerate (i.e. $k$ ) by changing a single independent variable, $P_{\mathrm{G} 2}$. For a given value of $\mathrm{P}_{\mathrm{G} 2}$ the value of $k_{\text {crit }}$ (i.e. the $k$ corresponding to point of voltage collapse) can be calculated using powerflow simulations with the optimal multiplier of [22]. Figure 6 plots these values of $k_{\text {crit }}$ vs values of $P_{\mathrm{G} 2}$. Note that the maximum value of $k_{\text {crit }}$ shown in figure 6 is the same as that shown in figure 4 thus confirming that system voltage security was indeed maximized.

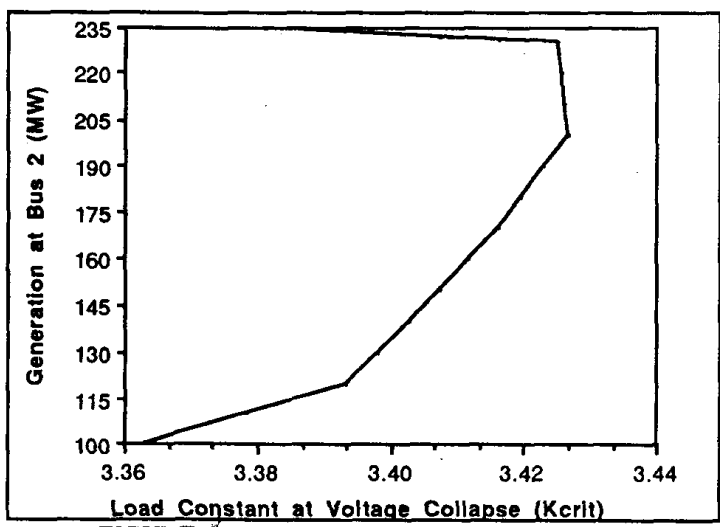

FIGURE 6: Load Constant at Collapse versus $P_{C_{2}}$

The New England 30 bus system was chosen as the second test system in order to demonstrate the method's usefulness on a larger system. The voltage collapse scenario is the same as that 
used in figure 1 and in [17], namely gradually increasing the reactive load at bus 11 . Here rather than a single controller as in the previous example, assume that the MW outputs of all the system generators are available for control (except Bus 30 which is the system slack). The first two columns of table 1 list these generators along with their respective basecase MW injections used in figure 1. The energy function can then be maximized for a given value of $Q_{11}$ (reactive load at bus 11) by using the algorithm mentioned in the beginning of this section. For $Q_{11}=800 \mathrm{MVAR}$ the initial sensitivities and the final "optimal" real power outputs of the generators are given in columns 3 and 4 of table 1 respectively. The associated change in the energy measure at this load level was from 3.71 to 4.66 . As was the case in figure 4, figure 7 plots the optimal energy versus the basepoint energy as $Q_{11}$ is varied. Note that $\mathrm{Q}_{11 \max }$ has increased from 1317MVAR to 1361MVAR.

\begin{tabular}{|lccc|}
\hline Gen & $\begin{array}{c}\text { Table 1 } \\
\text { Basecase } \\
\text { MW }\end{array}$ & $\begin{array}{c}\text { Sensitivity } \\
\mathrm{Q}_{11}=800 \mathrm{MVAR}\end{array}$ & $\begin{array}{c}\text { Optimal MW } \\
\mathrm{Q}_{11}=800 \mathrm{MVAR}\end{array}$ \\
2 & 250.0 & -0.032 & 582 \\
6 & 573.6 & -0.082 & 726 \\
10 & 650.0 & -0.305 & 111 \\
19 & 629.1 & -0.109 & 592 \\
20 & 505.5 & -0.101 & 603 \\
22 & 650.0 & -0.125 & 569 \\
23 & 558.6 & -0.124 & 510 \\
25 & 538.3 & -0.067 & 467 \\
29 & 824.8 & -0.094 & 713 \\
\hline
\end{tabular}

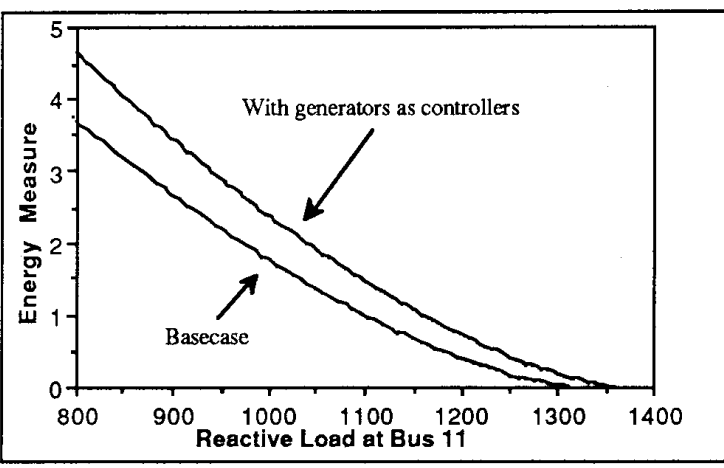

FIGURE 7: Optimal Security Enhancement with Redispatch

The goal of the previous algorithm was too maximize the energy measure for a given system load. However, for on-line use, where economic and other security constraints must be considered, the energy method sensitivities could be used in a different manner. For example, consider the constrained minimization problem where the objective is to minimize system operating costs such that no constraints are active (violating their limits). Linear programming optimization methods have proved effective in solving such power system problems [3],[23]. Voltage security could then be enforced by requiring that the energy for an area be above a specified threshold (perhaps determined by off-line studies). Any time the energy fell below that limit the constraint would be come active. Controller sensitivities would then be calculated using (10). These sensitivities would then simply form a row in the LP tableau.

\section{Calculation of Multiple Power Flow Solutions}

In order to apply energy methods to the voltage collapse problem, it is crucial that the appropriate unstable equilibrium (or low voltage) solutions can be found with reasonable computational effort. The calculation of the set of all UEP solutions for direct methods of transient stability has often been viewed as computationally prohibitive for large scale systems, due to an immense number of solutions. However, research on transient energy functions has shown that calculation of relevant UEPs can be quite tractable. In the voltage stability framework, the inclusion of the reactive power balance equations helps to diminish this problem significantly. In [16], [24] the practicality of locating relevant low voltage solutions on realistically sized networks (43 and 81 buses) was demonstrated using a rectangular NewtonRaphson technique with the optimal multiplier [21]. In addition, a "simplified method" is presented for calculating the up to N-1 (where $\mathbf{N}$ is the number of buses in the system) low voltage solutions corresponding to a low initial voltage guess at one bus.

The energy method, however, requires that only a single low voltage solution be found for those groups of buses in the power system which are judged vulnerable to voltage collapse. The appropriate solution in each area is typically characterized by depressed voltages localized within that area. Even in a large scale system the number of vulnerable areas is normally quite small and is not explicitly dependent upon network size. Experience of the authors with numerous test cases indicate that this set of low voltage solutions are a subset of the solutions calculated by the "simplified method" [25]. Furthermore the lowest energy measures were associated with solutions located simply by initializing a Newton-Raphson iteration (with optimal step size) initial guesses at the "weakest bus" within a weak area. Thus a heuristic algorithm can be employed to rapidly identify a subset of candidate low voltage solution buses. Since most low voltage solutions are local in nature, that is the voltages are only perturbed from the normal operating point in a subset of the system, the computational effort could be reduced by employing techniques found in [26] to only iterate on that portion of the system.

As the system moves towards voltage collapse the vulnerability of one area will normally dominate that of the others. References [23] and [27] show that the number of multiple solutions tends to decrease as system approaches voltage collapse. At the point immediately before collapse only a pair of closely located solutions exist. In [28] an algorithm is presented for locating the low voltage solution paired to the high voltage solution which requires computational time equivalent to that of a load flow. Thus the use of the energy method approach has the attractive property that as the system becomes more heavily loaded the computational burden actually goes down.

\section{Conclusions}

This paper has developed a method of reducing a system's vulnerability to voltage collapse by using the first order sensitivities of an energy function to controller changes. The paper first discussed the application of energy functions to the 
voltage collapse problem. Next an energy function was introduced and its controller sensitivities calculated. Then applicability of these sensitivities to increase the voltage security was demonstrated on both 5 and 30 bus systems. Lastly the computation aspects of the method were discussed, with emphasis on locating the appropriate low voltage solution.

The suitability of the controller sensitivities rests on the advantageous properties of the energy function. The energy function method offers a measure of proximity to voltage collapse which varies smoothly with changes in the system state. Also the measure is formulated in closed form and can be calculated with total computational requirements of only a few powerflow solutions. Additionally, the measure can be evaluated without the necessity of detailed projections about how the system parameters will change over time and is therefore insensitive to errors in these projections. The controller sensitivities provide an accurate measure of how controller movement will effect system voltage security and thus could be applied in a constrained optimization algorithm to increase system security.

\section{Acknowledgements}

The support of this work by the Electric Power Research Institute Project RP 2675-4 and National Science Foundation grant ECS-8857019 is gratefully acknowledged.

\section{References}

[1] A. Kurita and T. Sakurai, "The Power System Failure on July 23, 1987 in Tokyo", Proc. 27th IEEE Conf. Decision and Control, Austin, TX, Dec, 1988.

[2] North American Electric Reliability Council, 1987 System Disturbances, pp. 18, July 1988.

[3] T.J. Bertram, K.D. Demaree and L.C. Dangelmaier, "An Integrated Package for Real-Time Security Enhancement," PICA Proc., pp. 18-25, Seattle, WA, May 1989.

[4] M. Chau et. al., "Understanding Voltage Collapse in Bulk Transmission Systems", Engineering Foundation Conference on Bulk Power System Voltage Phenomena: Voltage Stability and Security, Potosi, MO, Sep. 1988.

[5] B.M. Weedy and B.R. Cox, "Voltage Stability of Radial Power Links," Proc. IEE, vol. 115, no. r, pp. 528-536, April 1968.

[6] E. Vaahedi and H. El-Din, "Considerations in Applying Optimal Power Flow to Power System Operation," IEEE Trans. on Power Systems, vol. PWRS-4, pp. 694-703, May 1989.

[7] C. Lemaitre et al., "An Indicator of the Risk of Voltage Profile Instability for Real-time Control Applications," IEEE PES Summer Meeting, SM 713-9, Long Beach, CA, July 1989.

[8] P. Kessel and H. Glavitsch, "Estimating Voltage Stability of a Power System," PICA Proc., pp. 424-430, May 1985.

[9] A. Tiranuchit and R.J. Thomas, "A Posturing Strategy against Voltage Instabilities in Electric Power Systems," IEEE Trans. on Power Systems, vol. PWRS-3, pp. 87-93, Feb., 1988.

[10] M.M. Begovic and A.G. Phadke, "Voltage Stability Assessment through Measurement of a Reduced State Vector," IEEE PES Summer Meeting, SM 709-7, Long Beach, CA, July 1989.

[11] Y. Tamura et al., "Current Issues in the Analysis of Voltage Instability Phenomena," Engineering Foundation Conference on Bulk Power System Voltage Phenomena: Voltage Stability and Security, Potosi, MO, Sep. 1988.

[12] A. Yokoyama and Y. Sekine, "A Static Voltage Stability Index Based on Multiple Load Flow Solutions," Engineering Foundation Conference on Bulk Power System Voltage Phenomena: Voltage Stability and Security, Potosi, MO, Sep. 1988.
[13] C.L. DeMarco and T.J. Overbye, "An Energy Based Security Measure for Assessing Vulnerability to Voltage Collapse," IEEE PES Summer Meeting, SM 712-1, Long Beach, CA, July 1989.

[14] C.L. DeMarco and A.R. Bergen, "A Security Measure for Random Load Disturbances in Nonlinear Power System Models," IEEE Trans. Circuits and Systems, pp. 1546-1557, vol. CAS-34, no. 12, Dec. 1987.

[15] A. Klos and A. Kemer, "The Non-Uniqueness of LoadFlow Solution", Proc. PSCC V, 3.1/8, Cambridge, 1975.

[16] Y. Tamura, K. Iba and S. Iwamoto, "A Method for Finding Multiple Load-Flow Solutions for General Power Systems", Proc. IEEE PES Winter Meeting, A80 043-0, New York, Feb. 1980.

[17] R.A. Schlueter et al.,"Voltage Stability and Security Assessment," EPRI Report, ÉL-5967, Proj. 1999-8, Aug. 1988.

[18] N. Narasimhumurthi and M. T. Musavi, "A General Energy Function for Transient Stability Analysis of Power Systems,"

IEEE Trans. Cir. and Sys, vol. CAS-31, pp. 637-645, July 1984.

[19] V. Vittal et al., "Derivation of Stability Limits using Analytical Sensitivity of the Transient Energy Margin," IEEE Winter Meeting, WM 207-2, New York, NY, Feb. 1989.

[20] W.F. Tinney, V. Brandwajn and S.M. Chan, "Sparse Vector Methods", IEEE Trans. Power App. Syst., Vol. PAS-104, No. 2, pp. 295-301, Feb. 1985

[21] G.W. Stagg and A.H. El-Abiad, Computer Methods in Power System Analysis, McGraw-Hill, New York, NY, 1968.

[22] S. Iwamoto and Y. Tamura, "A Load-Flow Calculation Method for Ill-Conditioned Power Systems," IEEE Trans. Power App. and Sys., vol PAS-100, pp. 1736-1743, April 1981.

[23] B. Stott and J.L. Marinho, "Linear Programming for Power System Security Applications," IEEE Trans. on Power App. and Sys., vol PAS-98, No. 3, pp. 837-848, May 1979.

[24] Y. Tamura, K. Sakamoto and Y. Tayama, "Voltage Instability Proximity Index (VIPI) Based on Multiple Load Flow Solutions in Ill-Conditioned Power Systems," Proc. 27th IEEE Conf. Decision and Control, Austin, TX, Dec. 1988.

[25] C.L. DeMarco and T.J. Overbye, "Low Voltage Power Flow Solutions and Their Role in Exit Time Base Security Measures for Voltage Collapse," Proc. 27th IEEE Conf. Decision and Control, Austin, TX, Dec. 1988.

[26] R. Bacher and W.F. Tinney, "Faster Local Power Flow Solutions: The Zero Mismatch Approach," IEEE Winter Meeting WM 129-8, New York, NY, Feb. 1989.

[27] Y. Tamura et al., "Monitoring and Control Strategies of Voltage Stability Based on Voltage Instability Index," Engineering Foundation Conference on Bulk Power System Voltage Phenomena: Voltage Stability and Security, Potosi, MO, Sep. 1988.

[28] K. Iba et al., "A Method for Finding a Pair of Multiple Load Flow Solutions in Bulk Power Systems," PICA Proc., pp. 98 104, Seattle, WA, May 1989.

Thomas J. Overbye (S '87) was born in Milwaukee, WI in 1960 He received his Bachelor of Science degree from the University of Wisconsin-Madison in 1983. He was employed with Madison Gas and Electric Company from 1983 through 1986. He is currently pursuing a $\mathrm{PhD}$ in Electrical Engineering at the University of Wisconsin-Madison.

Christopher L. DeMarco (S '80, M '85) was born in Derby, Connecticut in 1958. He received his Bachelor of Science degree in Electrical Engineering from the Massachusetts Institute of Technology in June of 1980, and his Ph.D. degree in Electrical Engineering and Computer Sciences from the University of California, Berkeley in May 1985. In January 1985, he joined the faculty of the Department of Electrical and Computer Engineering at the University of Wisconsin-Madison. 